Revue internationale d'éducation de Sèvres

\title{
Etats-Unis : les tests d'évaluation en débat
}

Cécile de Bouttemont

\section{(2) OpenEdition}

Journals

Édition électronique

URL : http://journals.openedition.org/ries/894

DOI : 10.4000/ries.894

ISSN : 2261-4265

Éditeur

Centre international d'études pédagogiques

Édition imprimée

Date de publication : 1 avril 2010

Pagination : 17-19

ISSN : 1254-4590

Référence électronique

Cécile de Bouttemont, «Etats-Unis : les tests d'évaluation en débat », Revue internationale d'éducation de Sèvres [En ligne], 53 | avril 2010, mis en ligne le 01 avril 2013, consulté le 24 avril 2019. URL : http:// journals.openedition.org/ries/894 ; DOI : 10.4000/ries.894

Ce document a été généré automatiquement le 24 avril 2019

(c) Tous droits réservés 


\title{
Etats-Unis : les tests d'évaluation en débat
}

\author{
Cécile de Bouttemont
}

1 La parution, en septembre et octobre 2009, de deux rapports mettant en cause l'un la qualité, l'autre le niveau des tests d'évaluation a relancé un débat déjà vif aux États-Unis. Le rapport du Government accountability Office $(\mathrm{GAO})$ a ainsi montré que, pour échapper aux coûts liés à la mise en place de tests de qualité, une majorité d'États augmente la proportion de tests QCM, moins onéreux que les tests à questions ouvertes. L'étude, publiée par le National Center for Education Statistics ${ }^{2}$ (NCES), met en évidence que, pour atteindre les objectifs officiels fixés par l'État fédéral, certains États abaissent le niveau de leurs tests.

2 La mise en place de tests d'évaluation uniformisés relève d'une politique plus générale d'évaluation mise en œuvre aux États-Unis dans les années quatre-vingts. L'évaluation standardisée est apparue alors comme l'une des solutions susceptibles d'améliorer tant le fonctionnement du système éducatif que les apprentissages des élèves. Cet objectif d'efficacité et d'équité a propulsé les tests d'évaluation au rang d'outil de pilotage du système éducatif alors que, traditionnellement, ils n'étaient qu'un instrument de mesure de la performance des élèves ${ }^{3}$. Cette évolution explique la vivacité d'un débat qui touche tous les acteurs de l'éducation aux États-Unis.

\section{Les tests d'évaluation : caractéristiques}

Dans un système totalement décentralisé, la diversité est de règle. Il n'existe pas d'examen national aux États-Unis: chacun des cinquante États élabore des tests pour évaluer la performance des élèves par rapport à des objectifs d'apprentissage ${ }^{4}$ qu'il a luimême définis. Le niveau de difficulté de ces tests et les standards sur lesquels ils s'appuient varient d'un État à l'autre. Les élèves sont testés annuellement en lecture et en mathématique de la classe trois à la classe huit puis en sciences au moins une fois par cycle depuis 2007-2008. Le NAEP (National Assessment of Educational Progress) est la seule 
évaluation nationale représentative des connaissances acquises dans différentes matières. Gérée au niveau fédéral, elle porte sur un échantillon représentatif de la population scolaire pour l'ensemble du territoire. Elle ne donne pas de résultats individuels, mais renseigne sur les scores de groupes d'élèves. Réalisée tous les deux ans, ses résultats sont publiés par l'État, par zone géographique ainsi que par type d'établissement scolaire, public et privé.

\section{Les tests d'évaluation au cœur du dispositif de pilotage}

4 L'obligation de justifier les dépenses que les gouvernements des États investissent en éducation n'est pas nouvelle ${ }^{5}$. Cependant, le nouveau dispositif établi en 2002 par la loi fédérale No Child Left Behind (NCLB) a renforcé et généralisé cette obligation. Les États risquent de ne plus bénéficier de crédits s'ils ne rendent pas compte de leurs performances annuelles en mathématiques et en lecture et s'ils ne mesurent pas les progrès réalisés selon les critères définis par le gouvernement fédéral. Cette loi fixe également de nouveaux objectifs en termes d'acquis des élèves, avec pour ambition que tous les élèves atteignent un niveau " avancé ${ }^{6}$ » en lecture et en mathématiques d'ici à 2014. Les établissements sont tenus de faire atteindre à leurs élèves un certain niveau de performance et/ou d'obtenir de meilleurs résultats d'une période d'évaluation à l'autre.

5 Les enjeux sont forts, tant pour les élèves que pour l'établissement : les élèves de vingthuit États doivent passer un test pour l'obtention du diplôme de fin d'études secondaires. Des recours sont prévus en cas de mauvaise performance de l'établissement. Ces recours vont du soutien technique à la mise sous tutelle si les équipes pédagogiques ne parviennent pas à inverser la tendance malgré l'aide reçue.

6 En contrepartie, l'établissement se voit octroyer une plus grande marge de manœuvre dans l'utilisation des fonds fédéraux et dans les moyens d'atteindre les objectifs; par ailleurs, l'équipe pédagogique est libre de choisir sa méthode et son rythme de progression.

\section{Les questions débattues autour du test d'évaluation}

7 Si le principe de l'évaluation standardisée semble généralement bien accepté aux ÉtatsUnis, il n'en reste pas moins que les modalités du système actuel font l'objet de vives critiques en termes de coût et de fiabilité : quarante-huit États sur les quarante-neuf interrogés indiquent, dans le rapport du GAO, se heurter à des problèmes de coût, de temps et ne pouvoir assurer la fiabilité des tests.

8 L'obligation d'amélioration annuelle des résultats scolaires oblige les États à mettre en place une série de tests qui s'avèrent coûteux dans leur conception comme dans leur administration. Ainsi une majorité d'entre eux remplacent les tests à questions ouvertes nécessitant un traitement plus long par des QCM moins coûteux à mettre en place. Enfin, certains États, plutôt que de les concevoir, recourent à des tests conçus par des sociétés privées, moins onéreux, au risque d'une inadéquation entre les tests et les standards étatiques. Pour atteindre l'objectif demandé, certains États abaissent le niveau des tests dans les matières fondamentales. D'après le rapport du NCES, trente-et-un États ont 
abaissé leurs exigences en lecture au-dessous du premier niveau de l'échelle et sept États l'ont abaissé en mathématiques pour la classe quatre.

La loi fédérale exige des États l'intégration dans leurs évaluations des élèves handicapés et des élèves maîtrisant mal l'anglais; or, trente-six États ont été cités par le gouvernement fédéral pour manquement à leurs obligations d'intégrer les élèves handicapés et trente-trois pour les élèves qui maîtrisent mal l'anglais?'.

Enfin, les conclusions du rapport du $\mathrm{CEP}^{8}$, entre autres, montrent l'impact significatif des tests d'évaluations sur le curriculum. Dans certaines classes, l'entraînement intensif aux tests d'évaluation se fait au détriment d'autres disciplines et selon l'importance des matières évaluées. Cependant, la focalisation sur les évaluations étatiques implique parfois un enrichissement du curriculum et l'évaluation standardisée donne des lignes directrices claires sur les contenus à enseigner, par ailleurs elle aide à mettre en évidence faiblesses et atouts des élèves, et constitue un cadre national cohérent ${ }^{9}$.

Le Président Obama vient d'annoncer un nouveau plan de relance du système éducatif, « Race to the top ", doté d'une enveloppe de quatre milliards de dollars. Elle sera répartie par le gouvernement fédéral entre les États qui adopteront les mesures souhaitées par le gouvernement. Parmi celles-ci, le gouvernement accorde une attention particulière à la mise en place de standards compétitifs, d'un meilleur système d'évaluation prenant en compte les compétences en plus des connaissances, d'un plan de formation ambitieux pour le recrutement de professeurs et de chefs d'établissement compétents, enfin d'un soutien plus important aux écoles les moins performantes.

\section{NOTES}

1. Cour des Comptes américaine. Il est disponible sur le site du GAO : http://www.gao.gov/ .

2. Disponible : http://nces.ed.gov/nationsreportcard/pubs/studies/2010456.asp/ .

3. Nathalie Mons, Les effets théoriques et réels de l'évaluation standardisée, Réseau Eurydice, 2009, $44 \mathrm{p}$.

4. Les standards de contenu.

5. Susan Fuhrman, «Vingt ans d'efforts pour une réforme de l'éducation », In : R. Normand, T. Bessy, J.-L. Derouet, Quelles politiques pour l'égalité ? Savoirs, gouvernances et obligation de résultats, INRP, 2009, p. 15.

6. L'échelle compte trois niveaux : basique, suffisant, avancé.

7. S. Fuhrman, p. 123.

8. CEP (Center on Education Policy), centre de recherche indépendant dont le rapport est accessible en ligne : http://www.cep-dc.org/ .

9. Nathalie Mons, ibid. p. 25. 
INDEX

Mots-clés : évaluation, système éducatif, test

Index géographique : États-Unis

\section{AUTEUR}

\section{CÉCILE DE BOUTTEMONT}

Documentaliste au Centre de ressources et d'ingénierie documentaires du Centre international d'études pédagogiques (CIEP). 